ORIGINAL ARTICLE

\title{
Identification of a plasma signature of psychotic disorder in children and adolescents from the Avon Longitudinal Study of Parents and Children (ALSPAC) cohort
}

\author{
A O'Gorman ${ }^{1,2}$, T Suvitaival ${ }^{3}$, L Ahonen ${ }^{3}$, M Cannon ${ }^{1}$, S Zammit ${ }^{4,5}$, G Lewis ${ }^{6}$, HM Roche , I Mattila $^{3}$, T Hyotylainen ${ }^{3,8}$, M Oresic $^{3,9,10}$, \\ L Brennan ${ }^{2,11}$ and DR Cotter ${ }^{1,11}$
}

The identification of an early biomarker of psychotic disorder is important as early treatment is associated with improved patient outcome. Metabolomic and lipidomic approaches in combination with multivariate statistical analysis were applied to identify plasma alterations in children (age 11) (38 cases vs 67 controls) and adolescents (age 18) (36 cases vs 117 controls) preceeding or coincident with the development of psychotic disorder (PD) at age 18 in the Avon Longitudinal Study of Parents and Children (ALSPAC). Overall, 179 lipids were identified at age 11, with 32 found to be significantly altered between the control and PD groups. Following correction for multiple comparisons, 8 of these lipids remained significant (lysophosphatidlycholines (LPCS) LPC(18:1), LPC(18:2), LPC(20:3); phosphatidlycholines (PCs) PC(32:2; PC(34:2), PC(36:4), PC(0-34-3) and sphingomyelin (SM) SM(d18:1/24:0)), all of which were elevated in the PD group. At age 18, 23 lipids were significantly different between the control and PD groups, although none remained significant following correction for multiple comparisons. In conclusion, the findings indicate that the lipidome is altered in the blood during childhood, long before the development of psychotic disorder. LPCs in particular are elevated in those who develop PD, indicating inflammatory abnormalities and altered phospholipid metabolism. These findings were not found at age 18, suggesting there may be ongoing alterations in the pathophysiological processes from prodrome to onset of PD.

Translational Psychiatry (2017) 7, e1240; doi:10.1038/tp.2017.211; published online 26 September 2017

\section{INTRODUCTION}

Psychotic disorders (PD) are among the most severe and debilitating medical diseases, with schizophrenia being the most common, affecting $\sim 0.5-1 \%$ of the global population. ${ }^{1}$ At present, the diagnosis of PD is subjective and there are no reliable biological diagnostic tests. ${ }^{2}$ Over the last decade, the psychosis field of research has shifted its focus to the prodrome or 'at-risk mental state' ('ARMS') in an attempt to identify and treat subjects at high risk of developing a psychotic illness. Investigations have indicated that $20-30 \%$ of these individuals will go on to develop schizophrenia over a 2-3 year period. ${ }^{3}$ Strong evidence supports early intervention as a means to alleviate and improve therapeutic outcome for individuals. ${ }^{4,5}$ Owing to the multifactorial complexity of $\mathrm{PD}^{2,6}$ the identification of a disease signature and molecular biomarker(s) sensitive to the underlying pathogenic factors would be of great importance to assist in early detection and diagnosis ${ }^{7}$ but also to understand the mechanisms underlying the disease process, which has yet to be fully elucidated. $^{8}$

Metabolomics, the global study of metabolites in cells, tissues or biofluids has emerged in recent years as a promising tool to identify abnormalities in psychotic illnesses. Metabolomic studies in schizophrenia and related psychoses have highlighted a number of metabolic perturbations such as glucoregulatory processes, ${ }^{9,10}$ fatty acid and lipid metabolism, ${ }^{11-13}$ mitochondrial function, ${ }^{14}$ and proline ${ }^{7}$ and tryptophan metabolism. ${ }^{15}$ Although a comprehensive mapping of disturbances in metabolic pathways in PD is still a long way off, ${ }^{16}$ the most consistent findings involve pathways common to fatty acids and the pro-oxidant/antioxidant balance. ${ }^{17} \mathrm{~A}$ recent study by Rice and colleagues reported decreased levels of erythrocyte polyunsaturated fatty acid (PUFA) levels in young people at ultra-high risk of $\mathrm{PD}^{18}$ providing additional evidence of the putative $\omega-3$ PUFA deficiency syndrome. These studies have identified various metabolite signatures and have contributed to a developing and enhanced understanding of the disease mechanism. However, they generally focus on the adult population who have already transitioned to schizophrenia, with a majority being medicated, and so they are limited in terms of identifying early biomarker signatures of the disease.

The aim of this study was to apply metabolomic approaches to identify plasma alterations in children (age 11) and adolescents

\footnotetext{
${ }^{1}$ Department of Psychiatry, Royal College of Surgeons in Ireland (RCSI), Beaumont Hospital, Dublin, Ireland; ${ }^{2}$ Institute of Food and Health, UCD School of Agriculture and Food Science, University College Dublin (UCD), Belfield, Dublin, Ireland; ${ }^{3}$ Steno Diabetes Center, Gentofte, Denmark; ${ }^{4}$ MRC Centre for Neuropsychiatric Genetics and Genomics, Cardiff University, Cardiff, UK; ${ }^{5}$ Centre for Academic Mental Health, School of Social \& Community Medicine, University of Bristol, Bristol, UK; ${ }^{6}$ Division of Psychiatry, University College London, London, UK; ${ }^{7}$ Nutrigenomics Research Group, UCD Conway Institute/UCD Institute of Food \& Health, School of Public Health, Physiotherapy \& Sports Science, University College Dublin (UCD), Belfield, Dublin, Ireland; ${ }^{8}$ Department of Chemistry, Örebro University, Örebro, Sweden; ${ }^{9}$ Turku Centre for Biotechnology, University of Turku and Åbo Akademi University, Turku, Finland and ${ }^{10}$ School of Medical Sciences, Örebro University, Örebro, Sweden. Correspondence: Professor L Brennan, Institute of Food and Health, UCD School of Agriculture and Food Science, University College Dublin (UCD), Belfield, Dublin 4, Ireland.
}

E-mail: Iorraine.brennan@ucd.ie or Professor DR Cotter, Department of Psychiatry, Royal College of Surgeons in Ireland (RCSI), Beaumont Hospital, Dublin 9, Ireland. E-mail: david.cotter@rcsi.ie

${ }^{11}$ These authors contributed equally to this study.

Received 3 March 2017; revised 2 August 2017; accepted 17 August 2017 
Table 1. An overview of the ALSPAC study population characteristics

\begin{tabular}{|c|c|c|c|c|}
\hline & $\begin{array}{l}\text { Controls age } 11 \\
\quad(n=67)\end{array}$ & $\begin{array}{c}\text { Cases age } 11 \\
(\mathrm{n}=38)\end{array}$ & $\begin{array}{c}\text { Controls age } 18 \\
\quad(\mathrm{n}=117)\end{array}$ & $\begin{array}{c}\text { Cases age } 18 \\
(\mathrm{n}=36)\end{array}$ \\
\hline $\begin{array}{l}\mathrm{BMI} \pm \text { s.d. } \\
\text { Male:female }\end{array}$ & $\begin{array}{c}17.73 \pm 2.53 \\
39 \mathrm{M}: 28 \mathrm{~F}\end{array}$ & $\begin{array}{c}18.2 \pm 3.36 \\
8 \mathrm{M}: 30 \mathrm{~F}\end{array}$ & $\begin{array}{c}22.69 \pm 3.51 \\
58 \mathrm{M}: 59 \mathrm{~F}\end{array}$ & $\begin{array}{c}23.12 \pm 3.74 \\
8 \mathrm{M}: 28 \mathrm{~F}\end{array}$ \\
\hline
\end{tabular}

(age 18) in the ALSPAC cohort who developed PD at age 18. Such alterations may serve as potential early metabolic signatures/ biomarkers.

\section{MATERIALS AND METHODS}

Study cohort

The study comprises individuals from the Avon Longitudinal Study of Parents and Children (ALSPAC) cohort. The ALSPAC cohort is a prospective general population cohort, and a rich resource of demographic, environmental and clinical data on the individuals involved. ${ }^{19}$ The initial cohort consisted of 14062 births. Written informed consent was obtained prior to taking the plasma samples. Ethical approval for the study was obtained from the ALSPAC Ethics and Law Committee and the Local Royal College of Surgeons in Ireland (RCSI) Research Ethics Committees. Please note that the study website contains details of all the data that are available through a fully searchable data dictionary (http://www.bristol.ac.uk/alspac/research ers/access).

Measures of psychotic experiences and psychotic disorder. Psychotic experiences (PE) were identified at 11 and 18 years through the face-toface, semi-structured Psychosis-Like Symptom (PLIKS) interview ${ }^{20}$ conducted by trained psychology graduates in assessment clinics, and were coded according to the definitions and rating rules for the Schedules for Clinical Assessment in Neuropsychiatry, Version 2.0 (Organisation 1994). Interviewers rated PEs as not present, suspected or definitely psychotic. Cases of psychotic disorder (PD) were defined as individuals with definite PEs that were not attributable to the effects of sleep or fever and when the PE occurred at least once per month over the past 6 months and caused severe distress, had a very negative effect on social/occupational function, or led to help seeking from a professional source. ${ }^{20}$ Control samples from age-matched individuals without suspected or definite PEs, or PD at age 18 were randomly selected to match cases (Table 1).

\section{Plasma availability in age 11 samples}

We identified 38 individuals with PD at age 18 (cases) who had plasma samples at age 11 available for the metabolomic/lipidomic analysis. Control samples $(n=67)$ from age and body mass index (BMI)-matched individuals without suspected or definite PEs at age 11 , or PD at age 18 were randomly selected to match cases (Table 1).

\section{Plasma availability in age 18 samples}

We identified 36 individuals with PD at age 18 (cases) who had plasma samples at age 18 available for the metabolomic/lipidomic analysis. Control samples $(n=117)$ from age and BMI-matched individuals who did not have PD at age 18 , or suspected or definite PEs at age 11 were randomly selected to match cases (Table 1). Of the PD cases, 25 individuals plasma samples were present at both age 11 and 18. Therefore, considering that there were 38 PD samples at age 11, and 36 PD samples at age 18, overall there were a total of 49 individual PD subjects studied.

\section{Plasma sample collection}

Non-fasting (age 11) and fasting (age 18) blood samples were collected from the participants into heparin Sarstedt S-Monovette tubes (Sarstedt, Numbrecht, Germany), stored on ice for a maximum of $90 \mathrm{~min}$ until processed. Post centrifugation, the plasma samples were stored at $-80^{\circ} \mathrm{C}$ until further analyses.

\section{Global lipidomic analysis}

The plasma samples were analysed for global profiling of lipids using a method developed specifically for lipidomics analyses. The samples were prepared following the previously published Folch procedure ${ }^{21}$ with minor modifications. Briefly, $10 \mu \mathrm{l}$ of $0.9 \% \mathrm{NaCl}, 100 \mu \mathrm{l}$ of $\mathrm{CHCl}_{3}: \mathrm{MeOH}(2: 1, \mathrm{v} / \mathrm{v})$ and $20 \mu \mathrm{l}$ of a $3.5 \mathrm{\mu g} \mathrm{ml}^{-1}$ working standard solution of chosen lipid standards (for quality control and data normalisation purposes) were added to $10 \mu \mathrm{l}$ of each plasma sample. The standard solution contained the following compounds: 1,2-diheptadecanoyl-sn-glycero-3-phosphoethanolamine ( $\mathrm{PE}(17: 0 / 17: 0)), \quad N$-heptadecanoyl-D-erythro-sphingosylphosphorylcholine $\quad(\mathrm{SM}(\mathrm{d} 18: 1 / 17: 0)), \quad N$-heptadecanoyl-D-erythrosphingosine (Cer(d18:1/17:0)), 1,2-diheptadecanoyl-sn-glycero-3-phosphocholine (PC(17:0/17:0)), 1-heptadecanoyl-2-hydroxy-sn-glycero-3-phosphocholine $(\mathrm{LPC}(17: 0))$ and 1-palmitoyl-d31-2-oleoyl-sn-glycero-3phosphocholine $(P C(16: 0 / d 31 / 18: 1))$. These were purchased from Avanti Polar Lipids (Alabaster, AL, USA). In addition, 1,2-dimyristoyl-sn-glycero-3phospho(choline- $d_{13}$ ) (PC(14:0/d13)) was purchased from Sigma Aldrich (Wicklow, Ireland) and Tripalmitin-1,1,1-13C3 (TG(16:0/16:0/16:0)-13C3) and Trioctanoin-1,1,1-13C3 (TG(8:0/8:0/8:0)-13C3) from Larodan (Solna, Sweden) for the same purpose. The samples were vortex mixed and allowed to stand on ice for $30 \mathrm{~min}$ after which they were centrifuged $\left(9400 \mathrm{~g}, 3 \mathrm{~min}, 4^{\circ} \mathrm{C}\right.$ ). Overall, $60 \mathrm{\mu l}$ from the lower layer of each sample was then transferred to a glass vial with an insert and $60 \mu \mathrm{l}$ of $\mathrm{CHCl}_{3}: \mathrm{MeOH}(2: 1$, $\mathrm{v} / \mathrm{v}$ ) was added. The samples were then stored at $-80^{\circ} \mathrm{C}$ until analysis.

Calibration curves (at concentration levels of 100, 500, 1000, 1500, 2000 and $2500 \mathrm{ng} \mathrm{ml}^{-1}$ ) for quantification of lipids were prepared using 1hexadecyl-2-(9Z-octadecenoyl)-sn-glycero-3-phosphocholine (PC(16:0e/ 18:1(9Z))), 1-(1Z-octadecenyl)-2-(9Z-octadecenoyl)-sn-glycero-3-phosphocholine (PC(18:0p/18:1(9Z))), 1-octadecanoyl-sn-glycero-3-phosphocholine (LPC(18:0)), 1-(1Z-octadecenyl)-2-docosahexaenoyl-sn-glycero-3-phosphocholine ( $\mathrm{PC}(18: 0 \mathrm{p} / 22$ :6)), 1-stearoyl-2-arachidonoyl-sn-glycero-3-phosphoinositol (PI(18:0/20:4)) and 1-stearoyl-2-linoleoyl-sn-glycerol (DG(18:0/20:4)) from Avanti Polar Lipids, 1-Palmitoyl-2-Hydroxy-sn-Glycero-3-Phosphatidylcholine ( $\operatorname{LPC}(16: 0))$ from Larodan, and 1,2,3-Triheptadecanoylglycerol (TG(17:0/17:0/17:0)) and 3ß-hydroxy-5-cholestene 3-linoleate (ChoE(18:2)) from Sigma Aldrich.

The samples were analysed using an ultra-high-performance liquid chromatography quadrupole time-of-flight mass spectrometry method (UHPLC-Q-TOF-MS). The UHPLC system was a 1290 Infinity system from Agilent Technologies (Santa Clara, CA, USA), which was equipped with a multisampler (maintained at $10^{\circ} \mathrm{C}$ ) using $10 \% \mathrm{DCM}$ in $\mathrm{MeOH}$ and $\mathrm{ACN}$ : MeOH:IPA: $\mathrm{H}_{2} \mathrm{O}(1: 1: 1: 1, \mathrm{v} / \mathrm{v} / \mathrm{v} / \mathrm{v})+0.1 \% \mathrm{HCOOH}$ as needle wash solutions after each injection for $7.5 \mathrm{~s}$ each, a quaternary solvent manager and a column thermostat (maintained at $50^{\circ} \mathrm{C}$ ). Separations were performed on an ACQUITY UPLC BEH C18 column $(2.1 \mathrm{~mm} \times 100 \mathrm{~mm}$, particle size $1.7 \mu \mathrm{m}$ ) by Waters (Milford, CT, USA). The flow rate was $0.4 \mathrm{ml} \mathrm{min}^{-1}$ and the injection volume was $1 \mu \mathrm{l} . \mathrm{H}_{2} \mathrm{O}+1 \% \mathrm{NH}_{4} \mathrm{Ac}(1 \mathrm{M})+0.1 \% \mathrm{HCOOH}(\mathrm{A})$ and ACN:IPA $(1: 1, v / v)+1 \% \mathrm{NH}_{4} \mathrm{Ac}+0.1 \% \mathrm{HCOOH}$ (B) were used as the mobile phases for the gradient elution. The gradient was as follows: from 0 to 2 min $35-80 \%$ B, from 2 to $7 \mathrm{~min} 80-100 \%$ B and from 7 to 14 min $100 \%$ B. Each run was followed by a $7 \mathrm{~min}$ re-equilibration period under initial conditions (35\% B). The mass spectrometer was a $6550 \mathrm{iFunnel}$ quadrupole time-of-flight (Q-TOF) from Agilent Technologies (Agilent) interfaced with a dual jet stream electrospray (dual ESI) ion source. Nitrogen generated by a nitrogen generator (PEAK Scientific, Renfrewshire, Scotland, UK) was used as the nebulising gas at a pressure of $21 \mathrm{psi}$, as the drying gas at a flow rate of $141 \mathrm{~min}^{-1}$ (at $193^{\circ} \mathrm{C}$ ) and as the sheath gas at a flow rate of $111 \mathrm{~min}^{-1}$ (at $379^{\circ} \mathrm{C}$ ). Pure nitrogen $(6.0)$ from Praxair (Fredericia, Denmark) was used as the collision gas. The capillary voltage and the nozzle voltage were kept at 3643 and $1500 \mathrm{~V}$, respectively. The reference mass solution including ions at $\mathrm{m} / \mathrm{z} 121.0509$ and 922.0098 was prepared according to instructions by Agilent and it was introduced to the mass spectrometer through the 
other nebuliser in the dual ESI ion source using a separate Agilent series 1290 isocratic pump at a constant flow rate of $4 \mathrm{ml} \mathrm{min}^{-1}$ (split to 1:100 before the nebuliser). The acquisition mass range was $m / z \quad 100-1700$ and the instrument was run using the extended dynamic range with an approximate resolution of $30000 \mathrm{FWHM}$ measured at $\mathrm{m} / \mathrm{z} 1521.9715$ (which is included in the tune mixture) during calibration of the instrument. MassHunters B.06.01 (Agilent) software was used for all data acquisition.

Quality control was performed throughout the dataset by including blanks, pure standard samples, extracted standard samples and control plasma samples. Relative standard deviations (\%RSDs) for retention times and peak areas for lipid standards representing each lipid class in the plasma samples and in the pure standard samples were calculated. The $\%$ RSDs for the retention times were on average $0.1 \%$ for both the plasma samples and for the pure standards samples. The \%RSDs for the peak areas were within accepted analytical limits at averages of 17.5 and $16.6 \%$ for the plasma samples and for the pure standard samples, respectively. This shows that the method is reliable and repeatable throughout the sample set.

Lipidomic data were pre-processed with MZmine 2 and peaks were identified based on the internal peak library. Peak areas were normalised by lipid class-specific internal standards and quantified with $\mathrm{R}$ based on the inverse-weighted linear model. Internal standard peaks were detected in a targeted way from the standards runs. The internal MS library's retention times were corrected with $\mathrm{R}$ to match the study with a linear correction based on the observed retention times in the standards runs. Other peaks in the sample runs were processed in a non-targeted way as: import of the mzML files, mass detection, chromatogram builder chromatogram deconvolution, isotopic peak grouper, peak filter, peak list row filter, join aligner, peak list row filter, gap filling, peak list filter, identification with custom data base search. The peak table was filtered in $\mathrm{R}$, allowing the peak to be missing in each of the eight batches in a maximum $50 \%$ of the samples. Remaining missing values were imputed with feature-wise half-the-minimum. All lipid metabolites, that were present (non-zero value) in more than $75 \%$ of samples were included in the data analyses.

\section{Global metabolomic analysis}

Polar metabolites were analysed in the plasma samples using comprehensive two-dimensional gas chromatography combined with time-offlight mass spectrometry $(\mathrm{GC} \times \mathrm{GC}-\mathrm{TOFMS}$, a LECO Pegasus 4D equipped with a cryogenic modulator from LECO, St. Joseph, MI, USA). Specifically, $400 \mu \mathrm{l}$ methanol and $10 \mu \mathrm{l}$ internal standard mixture heptadecanoic acid $\left(175 \mathrm{mg} \mathrm{I}^{-1}\right)$, DL-valine-d8 $\left(36 \mathrm{mg} \mathrm{I}^{-1}\right)$, succinic acid-d4 $\left(59 \mathrm{mg} \mathrm{I}^{-1}\right)$ and DLglutamic acid-d5 $\left(110 \mathrm{mg} \mathrm{I}^{-1}\right)$ were added to $30 \mu \mathrm{l}$ of plasma samples. The samples were vortex mixed and centrifuged for 5 min at 10000 r.p.m. and half of the supernatant was evaporated to dryness. This was followed by two step derivatisation using methoximation and silylation. Specifically, the samples were derivatised by adding first $25 \mu \mathrm{l}$ methoxamine $\operatorname{MOX}\left(45^{\circ} \mathrm{C}\right.$, $60 \mathrm{~min}$ ) and then $25 \mathrm{\mu l} \mathrm{N}$-trimethylsilyl- $\mathrm{N}$-methyl trifluoroacetamide MSTFA $\left(45^{\circ} \mathrm{C}, 60 \mathrm{~min}\right)$. Finally, $50 \mu \mathrm{l}$ of a retention index standard mixture $(n-$ alkanes, $\left.c=8 \mathrm{mg} \mathrm{I}^{-1}\right)$ with an injection standard (4,4'-dibromooctafluorobiphenyl, $\left.c=10 \mathrm{mg} \mathrm{I}^{-1}\right)$, both in hexane, was added to the mixture. The calibration consisted of six points for each quantified metabolite.

The columns were as follows: a methyl deactivated retention gap $(1.5 \mathrm{~m} \times 0.53 \mathrm{~mm}$ i.d.) was connected to $10 \mathrm{~m} \times 0.18 \mathrm{~mm}$ Rtx- 5 (i.d. diameter $0.18 \mathrm{~mm}$, df $f_{\text {phase }}$ thickness $0.20 \mu \mathrm{m}$ ) and to $1.5 \mathrm{~m} \times 0.1 \mathrm{~mm}$ BPX-50 (i.d. $0.1 \mathrm{~mm}$, df phase $_{\text {thickness }} 0.1 \mu \mathrm{m}$ ). Helium was used as the carrier gas at a constant pressure mode (40psig). A $4 \mathrm{~s}$ separation time was used in the second dimension. The temperature programme was as follows for the first-dimension column: $50^{\circ} \mathrm{C}(2 \mathrm{~min})$, at $7^{\circ} \mathrm{C} \mathrm{min}-1$ to $240^{\circ} \mathrm{C}$ and at $25^{\circ} \mathrm{C} \mathrm{min}-1$ to $300^{\circ} \mathrm{C}$ ( $3 \mathrm{~min}$ ). The second-dimension column temperature was $15^{\circ} \mathrm{C}$ higher than the corresponding firstdimension column throughout the programme.

The analytical method used allows for combined targeted and untargeted analysis, where a selected subset of metabolites can be quantified. In the present study, quantitation of 23 target metabolites (stearic acid, oleic acid, linoleic acid, palmitic acid, citric acid, glutamic acid, 3,4-dihydroxybutanoic acid, 3-hydroxybutyric acid, 2,4-dihydroxybutanoic acid, threonine, phenylalanine, serine, lactic acid, methionine, glycine, isoleucine, leucine, valine, proline, 2-hydroxybutyric acid, cholesterol, arachidonic acid and alanine) was done by external calibration curves for each individual metabolite.
ChromaTOF vendor software (LECO) was used for within-sample data processing, and the Guineu software (https://code.google.com/p/guineu/) was used for alignment, normalisation and peak matching across samples. ${ }^{22}$ The normalisation was performed by correction for internal standards and specific target metabolites were additionally quantified using external calibration curves. Other mass spectra from the GC $\times$ GCTOFMS analysis were searched against the NIST 14 Mass Spectral Library and Golm Metabolome Database, ${ }^{23}$ using also retention index data in the identification. Artefact peaks due to chemical background and compounds outside the linear range of the method were removed from the dataset. Control serum samples ( $n=32$ for human) and pure standards (to monitor the instrument performance and robustness) were analysed together with the samples. The relative standard deviation (RSD) for internal standards, spiked into the samples, was on average of $15.7 \%$ for the plasma samples. The RSD\% of the quantified metabolites in the control serum samples $(n=32)$ was on average $23.4 \%$. The RSD\% was $13.3 \%$ for isoleucine, $11.2 \%$ for leucine and $24.2 \%$ for valine in control serum samples $(n=4)$, and for internal standards in mice serum the RSD was on average $16.6 \%$. Neither sample preparation nor analysis order showed any significant effect on the results.

All plasma metabolite peaks that were present (non-zero value) in more than $75 \%$ of samples were included in the data analyses, including the unidentified ones. The unidentified peaks were annotated with their structural class from the Golm Metabolome Database using functional group prediction based on the fragmentation patterns. ${ }^{22,23}$

\section{Targeted metabolomic analysis}

A targeted metabolomic approach was taken to target a specific metabolic pathway, that is, the tricarboxylic acid (TCA) cycle (citric acid, succinic acid, fumaric acid, malic acid, a-ketoglutaric acid). A subset of control samples at both ages 11 and 18 were used for the analysis. This resulted in the following study numbers: PD at age 11 (38 controls vs 38 cases) and PD at age 18 (36 controls vs 36 cases) (Supplementary Table 4). Briefly, $50 \mu \mathrm{l}$ of $0.1 \mathrm{mg} \mathrm{ml}^{-1}$ of Labelled internal standards (succininc acid-2,2,3,3- $\mathrm{d}_{4}$, fumaric acid-2,3- $\mathrm{d}_{2}$, adipic acid-2,2,5,5- $\mathrm{d}_{4}$ and DL-malic acid-2,3,3- $\mathrm{d}_{3}$, Sigma Aldrich) were added to $100 \mu \mathrm{l}$ of plasma. To this, $440 \mu \mathrm{l}$ of anhydrous EtOH, $100 \mu \mathrm{l}$ pyridine, $500 \mu \mathrm{l}$ of deionised $\mathrm{H}_{2} \mathrm{O}$ and $150 \mu$ l of ethylchloroformate (ECF) was added and samples were vortexed. To this, $1000 \mu \mathrm{l}$ of $\mathrm{CHCl}_{3}$ was added. The $\mathrm{pH}$ was then adjusted to $5-6$ by adding $7 \mathrm{M} \mathrm{NaOH}$ solution and shaken for $5 \mathrm{~s}$. Overall, $100 \mu \mathrm{l}$ of ECF was added and vortexed for $20 \mathrm{~s}$. To this, $2 \mathrm{ml}$ of deionised $\mathrm{H}_{2} \mathrm{O}$ was added and vortexed. The samples were then centrifuged at $1700 \mathrm{~g}$ for $3 \mathrm{~min}$ at $4{ }^{\circ} \mathrm{C}$. The $\mathrm{CHCl}_{3}$ layer was transferred to a $10 \mathrm{ml}$ glass tube containing anhydrous sodium sulphate drying reagent. Following drying, samples were analysed using an Agilent 7200 Q-TOF GC/MS system (Agilent, Santa Clara, CA, USA).

Calibration was achieved by comparison of metabolite peak areas with reference to an external standard (TCA cycle metabolite library, Sigma Aldrich) and by comparison of their mass spectra with those in the NIST library (version 2.0). For quality control purposes, an aliquot from a pool of plasma was extracted and analysed in parallel with each batch of samples. Quantification of the TCA metabolites was carried out using the Masshunter TOF quantitative analysis software (version B.07.01, Agilent).

\section{Statistical analyses}

Censored regression analysis (SAS, version 9.3, Cary, NC, USA) was applied to the datasets to identify significant differences $(P<0.05)$ in lipid and metabolite levels between the control and PD groups at both age groups, adjusting for gender and BMI. Significant lipids and metabolites were corrected for multiple comparisons using the Benjamini-Hochberg step-up procedure in $\mathrm{R}$ (version 3.2.2) $(P<0.1)$. Censored regression analysis was applied as the majority of lipids and metabolite levels were not normaldistributed and were reported in varying concentration ranges among participants. Individual lipid levels were visualised using the beanplot algorithm implemented in R. A beanplot gives information on the mean lipid level within each group, the density of the data-point distribution and illustrates individual data points. ${ }^{11}$ Cluster analysis was performed using the Mclust package ${ }^{24}$ in $\mathrm{R}$ (version 3.2.2), which uses Gaussian mixture modelling fitted via the expectation-maximisation (EM) algorithm for model-based clustering. ${ }^{24}$ Prior to clustering, the data were normalised using a logarithmic transformation.

For the targeted metabolomic analysis, metabolite levels between groups were compared using general linear models gender and $\mathrm{BMI}$ as covariates (SPSS version 20, IBM, Armonk, NY, USA). 
Table 2. Differential plasma lipids between control and PD groups at age 11

\begin{tabular}{|c|c|c|c|c|}
\hline Lipid name & Control $(n=67)$ & Cases $(\mathrm{n}=38)$ & P-value & FDR-adjusted \\
\hline $\mathrm{CE}(18: 2)$ & $13481.42 \pm 4506.22$ & $15773.88 \pm 3728.61$ & 0.0392 & 0.242 \\
\hline CE(18:2)+unknown CE (667.6219) & $6568.58 \pm 2222.39$ & $7967.17 \pm 2181.29$ & 0.0313 & 0.2391 \\
\hline $\operatorname{LPC}(16: 1)$ & $38.27 \pm 5.84$ & $41.23 \pm 7.13$ & 0.0119 & 0.1776 \\
\hline LPC(18:1) & $231.84 \pm 63.24$ & $268.76 \pm 77.1$ & 0.0028 & 0.0972 \\
\hline LPC(18:2) & $394.75 \pm 128.23$ & $502.39 \pm 181.49$ & $<0.0001$ & 0.0179 \\
\hline LPC(20:3) & $37.21 \pm 6.83$ & $41.1 \pm 8.13$ & 0.0003 & 0.0269 \\
\hline $\operatorname{LPC}(20: 4)$ & $63.03 \pm 14.89$ & $66.84 \pm 15.93$ & 0.0246 & 0.2282 \\
\hline $\mathrm{PC}(16: 0 \mathrm{e} / 18: 1(9 \mathrm{Z}))$ & $92 \pm 29.94$ & $105.2 \pm 28.8$ & 0.0368 & 0.2391 \\
\hline $\mathrm{PC}(30: 0)$ & $56.88 \pm 16.51$ & $65.36 \pm 23.83$ & 0.0165 & 0.1981 \\
\hline$P C(32: 0)$ & $175.51 \pm 39.91$ & $200.61 \pm 38.49$ & 0.0083 & 0.1486 \\
\hline PC(32:1) & $238.88 \pm 90.72$ & $288.76 \pm 113.49$ & 0.0231 & 0.2282 \\
\hline $\mathrm{PC}(32: 2)$ & $61.56 \pm 20.85$ & $72.75 \pm 21.5$ & 0.0027 & 0.0972 \\
\hline PC(34:2) & $3759.47 \pm 717.83$ & $4257.93 \pm 741.34$ & 0.0065 & 0.0972 \\
\hline$P C(36: 2)$ & $2940.24 \pm 630.99$ & $3360.9 \pm 623.41$ & 0.0125 & 0.1776 \\
\hline PC(36:3) & $1753.26 \pm 669.47$ & $2152.76 \pm 569.66$ & 0.0349 & 0.2391 \\
\hline PC $(36: 4$ & $404.62 \pm 165.27$ & $504.24 \pm 198.32$ & 0.0038 & 0.0972 \\
\hline PC(38:2) & $70.5 \pm 22.97$ & $81.8 \pm 22.28$ & 0.0244 & 0.2282 \\
\hline PC(38:4) & $36.07 \pm 10.37$ & $40.94 \pm 10.62$ & 0.0374 & 0.2391 \\
\hline PC(40:6) & $56.74 \pm 20.41$ & $64.26 \pm 17.7$ & 0.0357 & 0.2391 \\
\hline $\mathrm{PC}(\mathrm{O}-32: 0)$ & $49.78 \pm 11.18$ & $55.84 \pm 11.43$ & 0.0078 & 0.1486 \\
\hline $\mathrm{PC}(\mathrm{O}-32: 1)$ & $37.88 \pm 7.19$ & $40.82 \pm 7.11$ & 0.0353 & 0.2391 \\
\hline $\mathrm{PC}(\mathrm{O}-34: 2)$ & $85.95 \pm 27.42$ & $98.13 \pm 30.17$ & 0.0255 & 0.2282 \\
\hline $\mathrm{PC}(\mathrm{O}-34: 3)$ & $111.62 \pm 33.27$ & $132.51 \pm 36.51$ & 0.0036 & 0.0972 \\
\hline $\mathrm{PC}(\mathrm{O}-36: 2)$ & $51.25 \pm 15.7$ & $57.48 \pm 14.77$ & 0.0166 & 0.1981 \\
\hline PC(O-36:3) & $42.86 \pm 11.11$ & $47.38 \pm 11.72$ & 0.0277 & 0.2361 \\
\hline $\mathrm{PC}(\mathrm{O}-38: 6)$ & $74.25 \pm 23.67$ & $84.11 \pm 22.81$ & 0.0455 & 0.2627 \\
\hline$S M(d 18: 0 / 16: 0)$ & $829.78 \pm 212.45$ & $933.75 \pm 194.41$ & 0.0129 & 0.1776 \\
\hline $\mathrm{SM}(\mathrm{d} 18: 1 / 24: 0)$ & $6167.04 \pm 2537.94$ & $7281.12 \pm 2418.58$ & 0.0029 & 0.0972 \\
\hline$S M(d 41: 1)$ & $3540.52 \pm 1381.32$ & $3889.15 \pm 1148.96$ & 0.0419 & 0.25 \\
\hline $\mathrm{SM}(\mathrm{d} 42: 2)$ & $15796 \pm 5875.87$ & $18218.69 \pm 5133.36$ & 0.0339 & 0.2391 \\
\hline TG(56:7) & $103.59 \pm 97.25$ & $76.17 \pm 46.64$ & 0.0198 & 0.2215 \\
\hline
\end{tabular}

Abbreviations: CE, cholesterol ester; LPC, lysophosphatidylcholine; PC, phosphatidylcholine; PD, psychotic disorder; SM, sphingomyelin; TG, triacylglycerol. Values are expressed as standard units \pm s.d. (standard deviation). $P$-values are adjusted for gender and BMI. FDR-adjusted $P$-values are adjusted for multiple comparisons using the Benjamini-Hochberg procedure. Values in bold are lipids that remain significant $(P<0.1)$ folllowing correction for multiple comparisons.

The levels of the lipids in the PD case group were analysed relative to the control group as a function of the acyl chain length and double bond content within each lipid group. The level ratio was computed by first computing each lipid's average level within each subject group, and the computing the ratio of these average levels between the PD case and the control groups. Each lipid's level ratio was shown on the $y$-axis of two lipid group-specific figures for each time point: one with the number of carbon atoms in the lipid's acyl chain on the $x$ axis and another with the number of double bonds in the lipids' acyl chain on the $x$ axis. These figures were used for the qualitative evaluation of changes in the lipids that could be related to the chain length and level of saturation.

\section{RESULTS}

The final lipidomic datasets used for analysis included a total of 179 lipids from the following lipid classes: cholesterol esters (CEs), lysophosphatidlycholines (LPCs), phosphatidlycholines (PCS), phosphatidylethanolamines (PEs), sphingomyelins (SMs), triglycerides (TGs), a diacylglycerol (DG) and a phosphatidylinositol (PI).

Age 11 and 18 lipidomic signatures of PD

At age 11 , a total of 32 lipids were significantly altered between controls and cases (nominal $P$-value $<0.05$ ). These lipids included three CEs, five LPCs, 19 PCs, four SM and a TG (Table 2). All of these lipids, with the exception of TG(56:7), were elevated in the PD group. Eight of these passed FDR significance $(P$-value $<0.1)$ (Figure 1). Relationships with the acyl chain length of lipid species was examined (Figures 2 and 3; Supplementary Figure 1). LPCs of lower carbon numbers and double bond content were higher in the PD group. An upward sloping relationship was seen in the mean ratio of SM levels in cases vs controls for higher carbon numbers. Clear patterns are not seen for the PCs and TGs, although the figure does highlight that the PCs are elevated in the PD group and TGs are downregulated, particularly so for those with a lower number of carbon double bonds.

The global lipidome was then evaluated by clustering the data into a set of lipid clusters. Cluster analysis identified seven lipid clusters (LC) and a description of each cluster is given in Table 3. Three of the identified clusters were significantly higher in the PD case group. LC4 was dominated by LPCs $(P$-value $=0.025)$, LC6 was dominated by both PCs and CEs $(P$-value $=0.036)$, whereas LC7 was a cluster containing PCs $(P$-value $=0.006)$.

At age 18, a total of 23 lipids were significantly different between the control and PD groups (Table 4; nominal $P$-value $<0.05$ ). These lipids were three LPCs, thirteen PCs, one PE, three SMs and three TGs. All of these lipids, with the exception of PC (36:4), were decreased in the PD group (Figure 2), although no clear pattern with respect to the acyl carbon chain length or double bond content was observed. The lipid cluster LC4 was significantly decreased in the PD group $(P$-value $=0.017)$ and was dominated by LPCs (Supplementary Table 1). None of these however passed significance at the selected FDR threshold of 0.1 .

Age 11 and 18 polar metabolomic signatures of PD

The metabolomic datasets used for analysis contained a total of 151 metabolites. At the age of 11, a total of 8 metabolites were significantly different between the control and PD groups, 6 of which were significantly decreased (1-monopalmitin, 2,3-dihydroxybutanoic acid, D-(-)-ribofuranose, ethanolamine, hydroxylamine and ribitol) and two significantly elevated (sugar derivatives) 

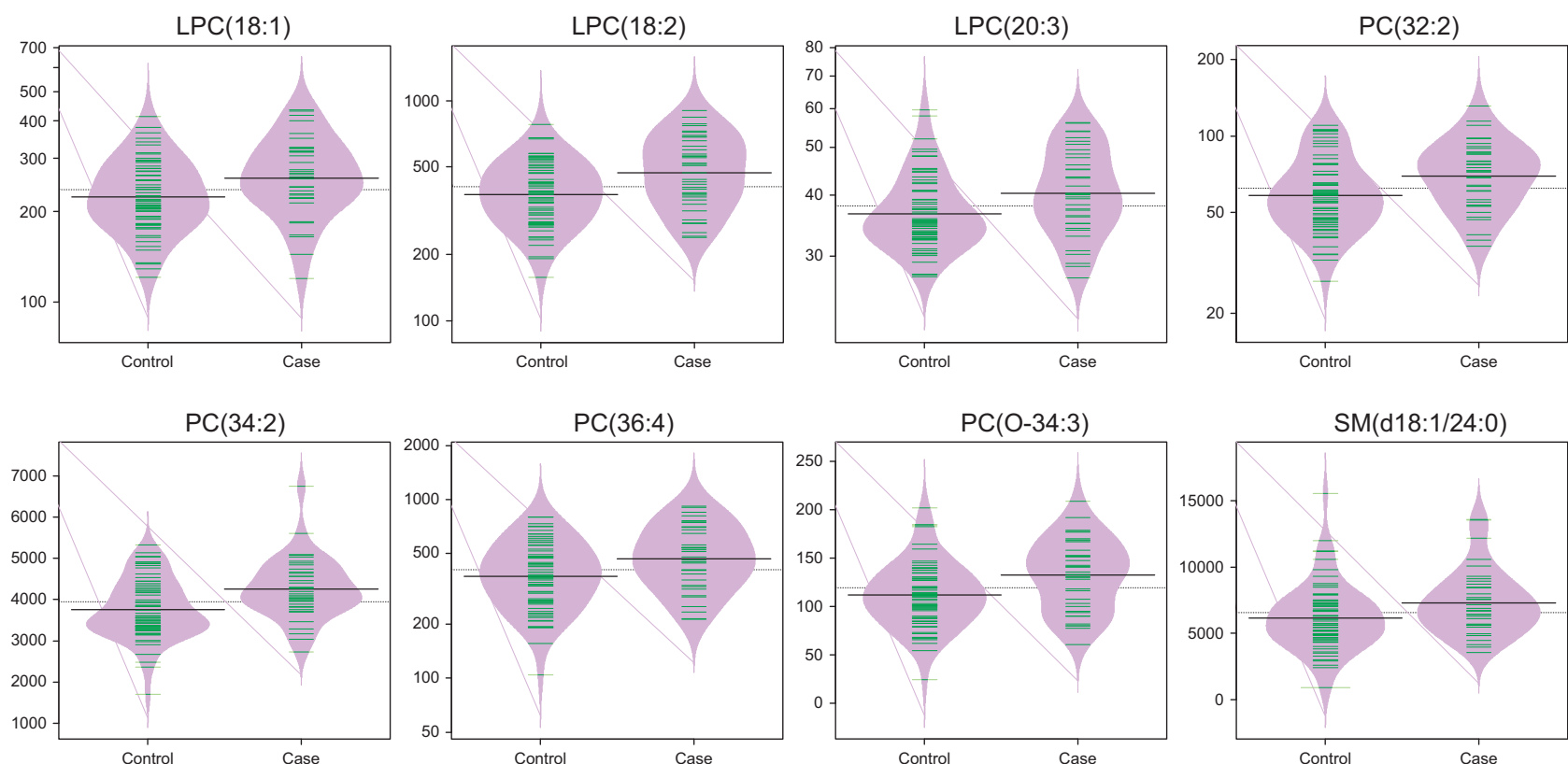

Figure 1. The levels of significant lipids at FDR level for age 11 samples.
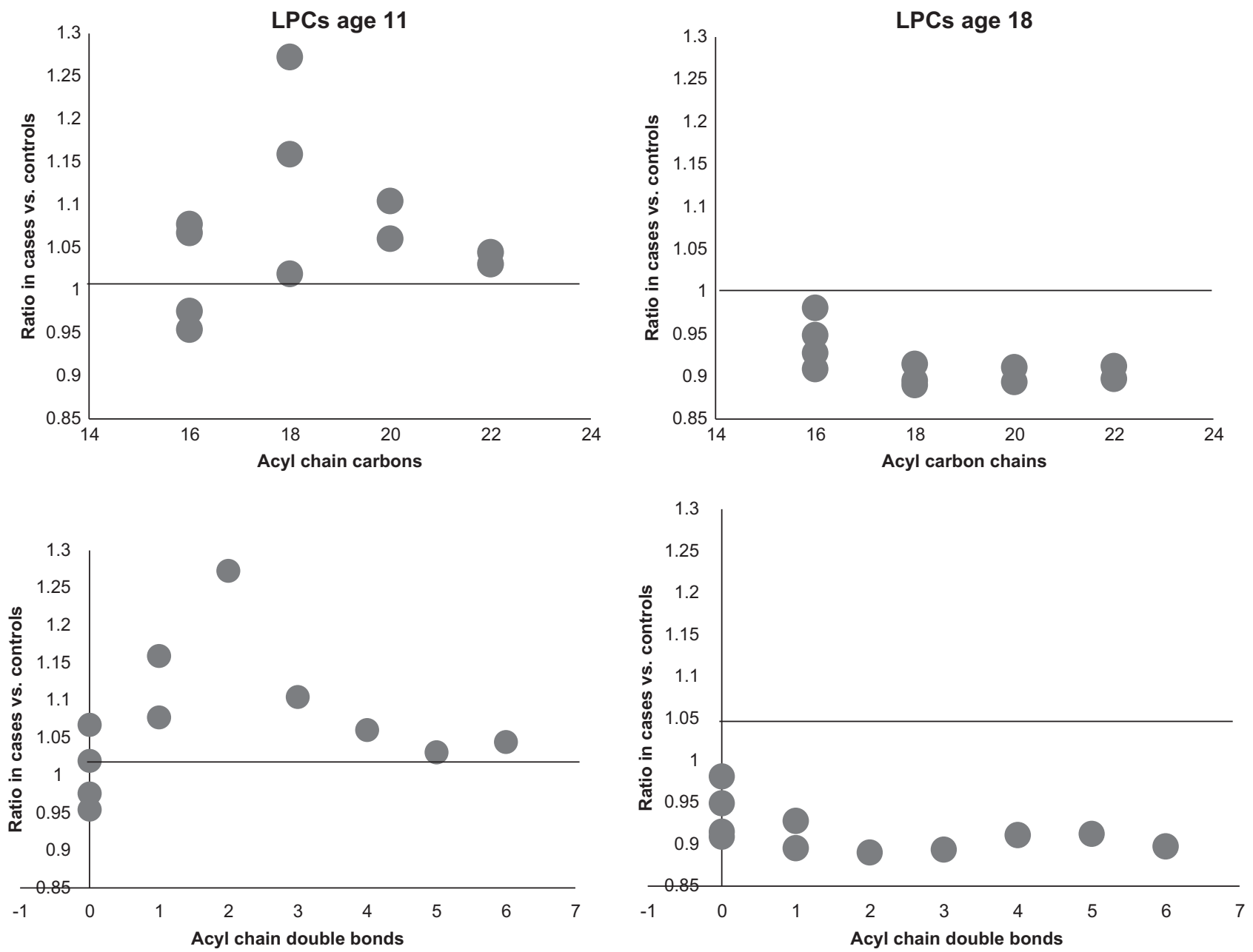

Figure 2. The relationship between psychotic disorder and acyl chain content in lipid species. The mean ratio of lipid levels in cases vs controls in plasma samples (ages 11 and 18) for lysophosphatidlycholines (LPCs). 

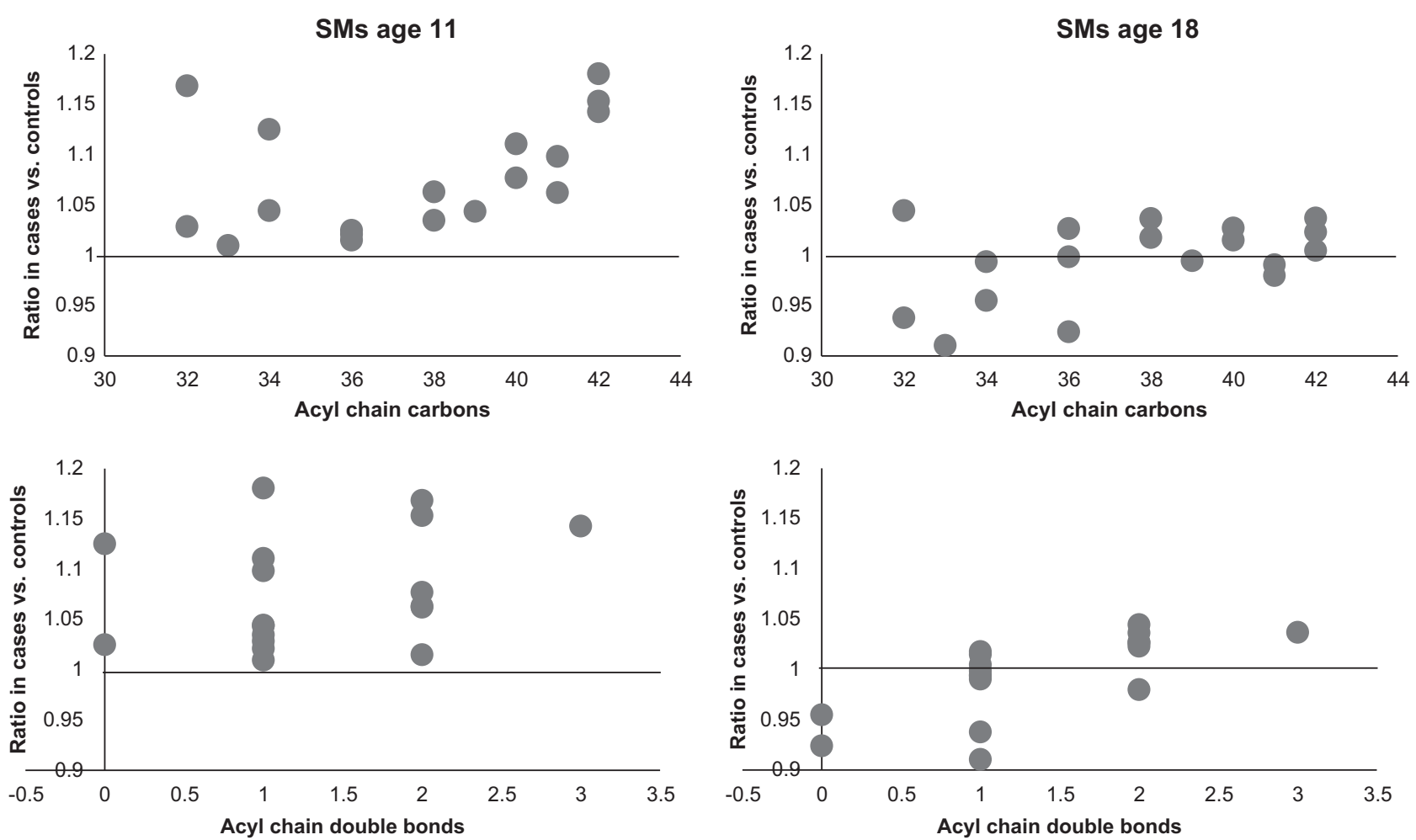

Figure 3. The relationship between psychotic disorder and acyl chain content in sphingomyelins (SMs). The mean ratio of lipid levels in cases vs controls in plasma samples (ages 11 and 18) for SMs.

Table 3. Description of lipid clusters obtained from lipidomics platform in the age 11 cohort and evaluated across the study groups

\begin{tabular}{lrll}
\hline Cluster name & Cluster size & Cluster description & $\begin{array}{c}\text { P-value (controls vs } \\
\text { cases) }\end{array}$ \\
\hline LC1 & 12 & TGs & 0.255 \\
LC2 & 44 & TGs & 0.966 \\
LC3 & 8 & LPCs & $\mathbf{0 . 0 2 5}$ \\
LC4 & 29 & PCs and TGs & 0.96 \\
LC5 & 23 & PCs and SM & 0.054 \\
LC6 & 40 & PCs and CEs & $\mathbf{0 . 0 3 6}$ \\
LC7 & 20 & PCs & $\mathbf{0 . 0 0 6}$ \\
\hline
\end{tabular}

Abbreviations: CEs, cholesterol esters; LC, lipid cluster; LPCs, lysophosphatidylcholines; PCs, phosphatidylcholines; SM, sphingomyelins; TGs, triacylclycerols. Values in bold are lipid clusters (LC) that are significant between controls vs cases $(P<0.05)$.

in the PD group (Table 5), although none were significant at the selected FDR threshold. Cluster analysis identified six metabolite clusters $(\mathrm{CL})$, none of which were significant between study groups (Supplementary Table 2).

At the age of 18, a total of 19 metabolites were significantly different between the case and control groups, none at FDR level (Table 6). These metabolites mainly belonged to the following classes of metabolites; organic acids, fatty acids and sugar/sugar derivatives and were all significantly decreased in the PD group with the exception of glycine and levoglucosan which were elevated in the PD group. 1-Monopalmitin is the only common significant metabolite between the studies and was found to be decreased in PD groups at both ages. The metabolite cluster CL1 was significantly decreased in the PD group $(P$-value $=0.034)$ and contained 22 metabolites consisting of fatty acids, organic acids and unknown metabolites (Supplementary Table 3).

Targeted analysis of the TCA cycle metabolites revealed no significant difference between the study groups at the ages of 11 and 18 . At the age of 18 , succinic acid was significantly decreased in the PD group $(P$-value $=0.04)$ (Table 7$)$.

\section{DISCUSSION}

Our findings indicate that the lipidome is altered between subjects with PD compared to subjects who do not experience any PEs, particularly at the age of 11 , giving promise to the potential of an early biomarker signature of PD. At the age of 11, a number of LPCs were found to be significantly elevated in the PD group. This is indicative of altered phospholipid metabolism, which has been considered as the pathophysiological basis of schizophrenia for some time. ${ }^{13}$ A number of studies have reported impaired phospholipid levels in schizophrenia subjects, although the specific results have not been consistent.

LPC, an inflammatory phospholipid generated by hydrolysis of PC via the actions of phospholipase $A_{2}\left(P L A_{2}\right)$, promotes inflammatory effects such as expression of endothelial cell adhesion molecules, growth factors, chemotaxis and activation of monocytes/macrophages. ${ }^{25}$ LPC has been suggested to have a functional role in the pathogenesis of various diseases including atherosclerosis, $^{26}$ diabetes $^{27}$ and systematic autoimmune diseases. $^{28}$ The association between inflammatory abnormalities and PD has been found repeatedly in many studies, which reported increased levels of pro-inflammatory compounds (mainly cytokines) in the serum, plasma and cerebrospinal fluid of patients with psychosis ${ }^{29}$ or who transition to schizophrenia from the atrisk mental state. ${ }^{30}$ In support of the inflammatory theory in relation to psychotic outcome in the ALSPAC cohort, Khandaker et al. $^{31}$ reported higher levels of the systematic inflammatory 
Table 4. Differential plasma lipids between control and PD groups at age 18

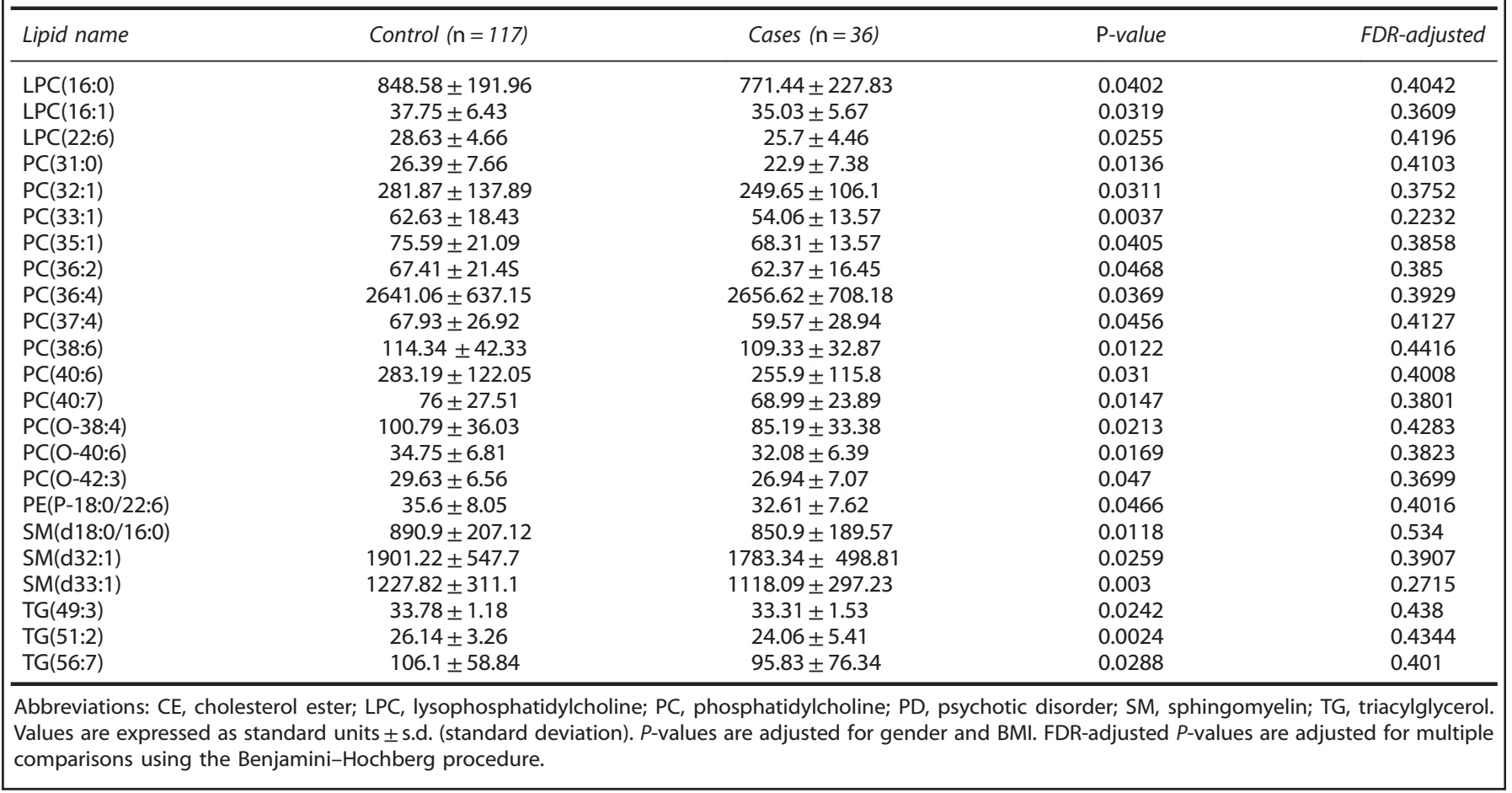

Table 5. Differential plasma metabolites between control and PD groups at age 11

\begin{tabular}{|c|c|c|c|c|}
\hline 1-monopalmitin & $448.89 \pm 162$ & $368.91 \pm 123.4$ & 0.0128 & 0.544 \\
\hline 2,4-Dihydroxybutanoic acid & $0.09 \pm 0.03$ & $0.07 \pm 0.02$ & 0.014 & 0.544 \\
\hline Ethanolamine & $250.51 \pm 88.88$ & $214.15 \pm 46.75$ & 0.0215 & 0.544 \\
\hline Ribitol & $175.9 \pm 41.79$ & $155.67 \pm 31.77$ & 0.0202 & 0.544 \\
\hline Hydroxylamine & $88.11 \pm 38.61$ & $66.9 \pm 39.02$ & 0.0043 & 0.544 \\
\hline Sugar derivative & $805.27 \pm 247.15$ & $961.92 \pm 337.63$ & 0.0274 & 0.591 \\
\hline
\end{tabular}

Abbreviations: BMl, body mass index; FDR, false discovery rate; PD, psychotic disorder. Values are expressed as standard units \pm s.d. (standard deviation). $P$-values are adjusted for gender and BMI. FDR-adjusted $P$-values are adjusted for multiple comparisons using the Benjamini-Hochberg procedure.

cytokine marker IL-6 in childhood, which was associated with an increased risk of developing psychosis in young adulthood.

Lipidomic studies have previously identified elevated plasma levels of LPCs (16:0, 18:0, 18:1 and 18:2) in first-episode neuroleptic naive schizophrenia patients as compared to healthy controls. ${ }^{12}$ Of the reported significant LPCs, two were also significantly elevated in this study at the age of 11 (LPC 18:1 and LPC 18:2), which suggests that LPCs with lower carbon numbers and fewer double bonds are associated with risk of PD. An earlier study reported increased platelet membrane LPC in schizophrenia patients. ${ }^{32}$ However, there are inconsistencies in studies, with one study reporting diminished levels of LPCs in the serum of schizophrenia patients compared to their co-twins as well as healthy controls. ${ }^{11}$ Furthermore, there are some noteworthy difference between this study and previous ones, particularly relating to the age of the subjects and the diagnosis. The samples studied in the current investigation were donated from participants at the ages 11 and 18 and were thus very different in terms of medication exposure and duration of psychotic illness from those of first episode or chronic schizophrenia. In comparison, the mean age of firstepisode schizophrenia patients in the study by Cai and colleagues was $27.5 \pm 9.5$ years $(n=11)$. In the twin study, which involved twin pairs discordant for schizophrenia, the participants had an average age of 51 years $(n=19)$, with the majority taking antipsychotic medication. The differences between these studies could explain some of the inconsistencies in the reported findings related to the direction of the LPCs and also highlights the need for validation studies for biomarker confirmation. Identification of the biomarkers at a young age prior to conversion to schizophrenia would be of more benefit clinically to assist early detection and diagnosis. ${ }^{7}$

A total of four PCs were found to be significantly elevated in the plasma of the PD group at age 11. At age 18, the PCs were decreased in the PD group, although not significant at the selected FDR threshold. A few studies have reported PCs to be related to psychotic outcome, with most of the studies reporting reduced levels in schizophrenia. ${ }^{33,34}$ The finding of elevated LPCs and PCs at age 11 and subsequent decreased levels relative to the controls (although not significant at FDR level) at age 18 suggest 
Table 6. Differential plasma metabolites between control and PD groups at age 18

\begin{tabular}{|c|c|c|c|c|}
\hline Metabolite name & Control $(n=117)$ & Cases $(n=36)$ & P-value & FDR-adjusted \\
\hline 1-Monopalmitin & $312.8 \pm 129.67$ & $254.3 \pm 112.61$ & 0.012 & 0.323 \\
\hline 2-Hydroxybutyric acid & $577.68 \pm 232.1$ & $489.04 \pm 213.93$ & 0.0161 & 0.323 \\
\hline 3-Hydroxybutyric acid & $36.64 \pm 37.35$ & $25.76 \pm 23.78$ & 0.0464 & 0.369 \\
\hline Cholesterol & $359.7 \pm 82.18$ & $335.36 \pm 75.08$ & 0.0165 & 0.323 \\
\hline Citric acid & $7.22 \pm 2.98$ & $5.85 \pm 2.36$ & 0.0329 & 0.346 \\
\hline Glycine & $11.52 \pm 3.19$ & $12.07 \pm 3.79$ & 0.0374 & 0.346 \\
\hline Leucine & $13.13 \pm 3.34$ & $11.23 \pm 2.72$ & 0.412 & 0.346 \\
\hline L-tryptophan & $14.95 \pm 7.76$ & $12.12 \pm 8.77$ & 0.0411 & 0.346 \\
\hline Oleic acid & $88.13 \pm 30.34$ & $79.9 \pm 25.78$ & 0.0344 & 0.346 \\
\hline Palmitic acid & $105.31 \pm 23.19$ & $97.2 \pm 16.08$ & 0.0171 & 0.323 \\
\hline Scyllo-inositol & $79.11 \pm 102.3$ & $51.76 \pm 40.75$ & 0.0215 & 0.325 \\
\hline Stearic acid & $35.94 \pm 8.4$ & $32.93 \pm 6.74$ & 0.0275 & 0.346 \\
\hline Sugar derivative & $1666.31 \pm 517.3$ & $1474.28 \pm 530.76$ & 0.0288 & 0.346 \\
\hline A144004 & $416 \pm 242.81$ & $314.94 \pm 301.41$ & 0.0125 & 0.323 \\
\hline Sugar derivative & $1253.88 \pm 362.07$ & $1076.74 \pm 395.7$ & 0.0137 & 0.323 \\
\hline Threonic acid & $1457.44 \pm 491.45$ & $1294.07 \pm 599.63$ & 0.02 & 0.325 \\
\hline Sugar derivative & $916.23 \pm 283.49$ & $840.44 \pm 324.57$ & 0.0155 & 0.323 \\
\hline Tocopherol, $\alpha$ & $453.34 \pm 307.71$ & $353.09 \pm 133.91$ & 0.0151 & 0.323 \\
\hline Levoglucosan & $31.55 \pm 52.17$ & $36.39 \pm 51.9$ & 0.0356 & 0.346 \\
\hline
\end{tabular}

Abbreviations: BMI, body mass index; FDR, false discovery rate; PD, psychotic disorder. Values are expressed as standard units \pm s.d. (standard deviation). Pvalues are adjusted for gender and BMI. FDR-adjusted $P$-values are adjusted for multiple comparisons using the Benjamini-Hochberg procedure.

\begin{tabular}{lccc}
\hline $\begin{array}{l}\text { Table 7. TCA cycle metabolite differences between study groups at } \\
\text { age } 18\end{array}$ & $\begin{array}{c}\text { Controls }(\mathrm{n}=36) \\
\left(\mathrm{mg} \mathrm{l}^{-1}\right)\end{array}$ & $\begin{array}{c}\text { Cases }(\mathrm{n}=36) \\
\left(\mathrm{mg} \mathrm{I}^{-1}\right)\end{array}$ & P-value \\
\hline Metabolite & $11.68 \pm 5.86$ & $9.43 \pm 4.84$ & 0.07 \\
\hline Citric acid & $0.57 \pm 0.24$ & $0.46 \pm 0.23$ & 0.04 \\
Succinic acid & $0.72 \pm 0.08$ & $0.77 \pm 0.48$ & 0.69 \\
Fumaric acid & $1.06 \pm 0.65$ & $0.87 \pm 0.68$ & 0.24 \\
a-ketoglutaric acid & $0.47 \pm 0.47$ & $0.52 \pm 0.47$ & 0.36 \\
Malic acid &
\end{tabular}

Abbreviations: BMl, body mass index; TCA, tricarboxylic acid. $P$-values are adjusted for gender and BMI.

that biomarker profiles may change with age. Furthermore, it suggests that the pathophysiological process can alter over time and with proximity to disease onset. ${ }^{35}$ A similar finding was reported in type 1 diabetes where increased LPCs were identified in children who later progressed to the disease. Following seroconversion to autoantibody positivity, the metabolic profiles of the participants were partially normalised, indicating that early biomarkers may be useful in diabetes prevention strategies. ${ }^{36}$ It has been suggested elsewhere that maybe the ideal biomarkers are involved in aetiological pathways and are subsequently likely to change according to disease status. ${ }^{37}$

In terms of the metabolome, very few metabolite differences were identified between control and PD groups (ages 11 and 18), with none significant at FDR level. 1-monopalmitin was reported to be decreased in the PD group at both ages and is a lipid implicated in membrane integrity and stability and an energy storage source. ${ }^{38}$ Citric acid and succinic acid (targeted metabolomic approach) were found to be reduced in the PD group at age 18. A number of previous studies have reported citric/citrate acid levels to be reduced in serum and urine samples in schizophrenia patients, with one study finding elevated levels in serum in a schizophrenia cohort, indicating a compromised energy metabolism. ${ }^{12,16}$ Decreased levels of citric acid and succinic acid indicate a dysfunction in TCA cycle activity and a deficit in mitochondrial respiration. ${ }^{16} \mathrm{~A}$ study comparing healthy controls vs schizophrenia pre-frontal cortex tissue reported that aerobic energy pathways were significantly decreased with reduced protein and transcript expression of TCA and oxidative phosphorylation enzymes, ${ }^{14}$ confirming compromised energy metabolism in individuals with schizophrenia. ${ }^{39-41}$

A major strength of this investigation is the ALSPAC cohort upon which it was based. The ALSPAC cohort includes detailed longitudinal clinical assessments and biosampling. The plasma samples were collected at age 11, prior to the development of psychotic disorder, and were not subject to confounds of drug exposure and chronic illness. Our observation of an altered lipidome at age 11, therefore, highlights a perturbed metabolism related to inflammation in the PD group, which may be an early biomarker signature of the disease, with the LPCs representing potential future early biomarkers of PD. The detected associations indicate that lipidomic profiles are useful for identifying important disease signatures. However, as a step to move these signatures closer to utilisation in the clinic validation in an external cohort is necessary. Notwithstanding this, the present study is an important step towards the identification of a signature associated with the development of PD.

\section{CONFLICT OF INTEREST}

The authors declare no conflict of interest.

\section{ACKNOWLEDGMENTS}

We are extremely grateful to all the families who took part in this study, the midwives for their help in recruiting them, and the whole ALSPAC team, which includes interviewers, computer and laboratory technicians, clerical workers, research scientists, volunteers, managers, receptionists and nurses. The UK Medical Research Council and Wellcome (Grant Ref: 102215/2/13/2) and the University of Bristol provide core support for ALSPAC. This study is also supported by the NIHR Bristol BRC. This research was specifically funded by the Irish Health Research Board through a Clinician Scientist Award to DRC. This project was funded by the Health Research Board (ref HRA-POR-2013-282; HRB CSA 2012/8). LB is supported by a European Research Council grant (ERC (647783)). We thank Anette Untermann at Steno Diabetes Center A/S for preparing all samples for the lipidomics analyses and for the polar metabolomics analyses.

\section{DISCLAIMER}

This publication is the work of the authors and will serve as guarantors for the contents of this paper. 


\section{REFERENCES}

1 Ustun TB. The global burden of mental disorders. Am J Public Health 1999; 89: 1315-1318.

2 van Os J, Kapur S. Schizophrenia. Lancet 2009; 374: 635-645.

3 Fusar-Poli P, Bonoldi I, Yung AR, Borgwardt S, Kempton MJ, Valmaggia L et al. Predicting psychosis: meta-analysis of transition outcomes in individuals at high clinical risk. Arch Gen Psychiatry 2012; 69: 220-229.

4 Larsen TK, Melle I, Auestad B, Haahr U, Joa I, Johannessen JO et al. Early detection of psychosis: positive effects on 5-year outcome. Psychol Med 2011; 41: 1461-1469.

5 Addington J, Cornblatt BA, Cadenhead KS, Cannon TD, McGlashan TH, Perkins DO et al. At clinical high risk for psychosis: outcome for nonconverters. Am J Psychiatry 2011; 168: 800-805.

6 Sawa A, Snyder SH. Schizophrenia: diverse approaches to a complex disease. Science 2002; 296: 692-695.

7 Oresic M, Tang J, Seppanen-Laakso T, Mattila I, Saarni SE, Saarni SI et al. Metabolome in schizophrenia and other psychotic disorders: a general populationbased study. Genome Med 2011; 3: 19.

8 Yang J, Chen T, Sun L, Zhao Z, Qi X, Zhou K et al. Potential metabolite markers of schizophrenia. Mol Psychiatry 2013; 18: 67-78.

9 Holmes E, Tsang TM, Huang JT, Leweke FM, Koethe D, Gerth CW et al. Metabolic profiling of CSF: evidence that early intervention may impact on disease progression and outcome in schizophrenia. PLoS Med 2006; 3: e327.

10 Schwarz E, Prabakaran S, Whitfield P, Major H, Leweke FM, Koethe D et al. High throughput lipidomic profiling of schizophrenia and bipolar disorder brain tissue reveals alterations of free fatty acids, phosphatidylcholines, and ceramides. J Proteome Res 2008; 7: 4266-4277.

11 Oresic M, Seppanen-Laakso T, Sun D, Tang J, Therman S, Viehman R et al. Phospholipids and insulin resistance in psychosis: a lipidomics study of twin pairs discordant for schizophrenia. Genome Med 2012; 4: 1.

12 Cai HL, Li HD, Yan XZ, Sun B, Zhang Q, Yan M et al. Metabolomic analysis of biochemical changes in the plasma and urine of first-episode neuroleptic-naive schizophrenia patients after treatment with risperidone. J Proteome Res 2012; 11: 4338-4350.

13 Khan MM, Evans DR, Gunna V, Scheffer RE, Parikh VV, Mahadik SP. Reduced erythrocyte membrane essential fatty acids and increased lipid peroxides in schizophrenia at the never-medicated first-episode of psychosis and after years of treatment with antipsychotics. Schizophr Res 2002; 58: 1-10.

14 Prabakaran S, Swatton JE, Ryan MM, Huffaker SJ, Huang JT, Griffin JL et al. Mitochondrial dysfunction in schizophrenia: evidence for compromised brain metabolism and oxidative stress. Mol Psychiatry 2004; 9: 684-697, 643.

15 Yao JK, Dougherty GG Jr., Reddy RD, Keshavan MS, Montrose DM, Matson WR et al. Altered interactions of tryptophan metabolites in first-episode neurolepticnaive patients with schizophrenia. Mol Psychiatry 2010; 15: 938-953.

16 Xuan J, Pan G, Qiu Y, Yang L, Su M, Liu Y et al. Metabolomic profiling to identify potential serum biomarkers for schizophrenia and risperidone action. J Proteome Res 2011; 10: 5433-5443.

17 Money TT, Bousman CA. Metabolomics of psychotic disorders. Metabolomics 2013; 3: 117.

18 Rice SM, Schafer MR, Klier C, Mossaheb N, Vijayakumar N, Amminger GP. Erythrocyte polyunsaturated fatty acid levels in young people at ultra-high risk for psychotic disorder and healthy adolescent controls. Psychiatry Res 2015; 228: 174-176.

19 Boyd A, Golding J, Macleod J, Lawlor DA, Fraser A, Henderson J et al. Cohort Profile: the 'children of the 90s'-the index offspring of the Avon Longitudinal Study of Parents and Children. Int J Epidemiol 2013; 42: 111-127.

20 Zammit S, Kounali D, Cannon M, David AS, Gunnell D, Heron J et al. Psychotic experiences and psychotic disorders at age 18 in relation to psychotic experiences at age 12 in a longitudinal population-based cohort study. Am J Psychiatry 2003; 170: 742-750.

21 Folch J, Lees M, Sloane Stanley GH. A simple method for the isolation and purification of total lipides from animal tissues. J Biol Chem 1957; 226: 497-509.

22 Castillo S, Mattila I, Miettinen J, Oresic M, Hyotylainen T. Data analysis tool for comprehensive two-dimensional gas chromatography/time-of-flight mass spectrometry. Anal Chem 2011; 83: 3058-3067.
23 Kopka J, Schauer N, Krueger S, Birkemeyer C, Usadel B, Bergmuller E et al. GMD@CSB.DB: the Golm Metabolome Database. Bioinformatics 2005; 21: 1635-1638.

24 Fraley C, Raftery AE. Model-based methods of classification: using the mclust software in chemometrics. J Stat Softw 2007; 18: 1-13.

25 Kita T, Kume N, Ishii K, Horiuchi $\mathrm{H}$, Arai $\mathrm{H}$, Yokode M. Oxidized LDL and expression of monocyte adhesion molecules. Diabet Res Clin Pract 1999; 45: 123-126.

26 Lusis AJ. Atherosclerosis. Nature 2000; 407: 233-241.

27 Shi AH, Yoshinari M, Wakisaka M, Iwase M, Fujishima M. Lysophosphatidylcholine molecular species in low density lipoprotein of type 2 diabetes. Horm Metab Res 1999; 31: 283-286.

28 Wu R, Svenungsson E, Gunnarsson I, Andersson B, Lundberg I, Schafer Elinder L et al. Antibodies against lysophosphatidylcholine and oxidized LDL in patients with SLE. Lupus 1999; 8: 142-150.

29 Bergink V, Gibney SM, Drexhage HA. Autoimmunity, inflammation, and psychosis: a search for peripheral markers. Biol Psychiatry 2014; 75: 324-331.

30 Focking M, Dicker P, Lopez LM, Cannon M, Schafer MR, McGorry PD et al. Differential expression of the inflammation marker IL12p40 in the at-risk mental state for psychosis: a predictor of transition to psychotic disorder? BMC Psychiatry 2016; 16: 326 .

31 Khandaker GM, Pearson RM, Zammit S, Lewis G, Jones PB. Association of serum interleukin 6 and C-reactive protein in childhood with depression and psychosis in young adult life a population-based longitudinal study. JAMA Psychiatry 2014; 71: 1121-1128.

32 Pangerl AM, Steudle A, Jaroni HW, Rufer R, Gattaz WF. Increased platelet membrane lysophosphatidylcholine in schizophrenia. Biol Psychiatry 1991; 30: 837-840.

33 Ryazantseva NV, Novitskii VV, Kublinskaya MM. Changes in the lipid phase of erythrocyte membranes in patients with paranoid schizophrenia. Bull Exp Biol Med 2002; 133: 84-86.

34 Kaddurah-Daouk R, McEvoy J, Baillie R, Zhu H, J KY, Nimgaonkar VL et al. Impaired plasmalogens in patients with schizophrenia. Psychiatry Res 2012; 198: 347-352.

35 Courvoisie H, Labellarte MJ, Riddle MA. Psychosis in children: diagnosis and treatment. Dialogues Clin Neurosci 2001; 3: 79-92.

36 Oresic M, Simell S, Sysi-Aho M, Nanto-Salonen K, Seppanen-Laakso T, Parikka V et al. Dysregulation of lipid and amino acid metabolism precedes islet autoimmunity in children who later progress to type 1 diabetes. $J$ Exp Med 2008; 205: 2975-2984.

37 Chana G, Bousman CA, Money T, Gibbons A, Gillett P, Dean B et al. Biomarker investigations related to pathophysiological pathways in schizophrenia and psychosis. Front Cell Neurosci 2013; 7: 95.

38 Motsinger-Reif AA, Zhu H, Kling MA, Matson W, Sharma S, Fiehn O et al. Comparing metabolomic and pathologic biomarkers alone and in combination for discriminating Alzheimer's disease from normal cognitive aging. Acta Neuropathol Commun 2013; 1: 28.

39 Pennington K, Beasley CL, Dicker P, Fagan A, English J, Pariante CM et al. Prominent synaptic and metabolic abnormalities revealed by proteomic analysis of the dorsolateral prefrontal cortex in schizophrenia and bipolar disorder. Mol Psychiatry 2008; 13: 1102-1117.

40 Focking M, Lopez LM, English JA, Dicker P, Wolff A, Brindley E et al. Proteomic and genomic evidence implicates the postsynaptic density in schizophrenia. Mol Psychiatry 2015; 20: 424-432.

41 English JA, Pennington K, Dunn MJ, Cotter DR. The neuroproteomics of schizophrenia. Biol Psychiatry 2011; 69: 163-172.

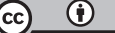

This work is licensed under a Creative Commons Attribution 4.0 International License. The images or other third party material in this article are included in the article's Creative Commons license, unless indicated otherwise in the credit line; if the material is not included under the Creative Commons license, users will need to obtain permission from the license holder to reproduce the material. To view a copy of this license, visit http://creativecommons.org/licenses/ by/4.0/

(c) The Author(s) 2017

Supplementary Information accompanies the paper on the Translational Psychiatry website (http://www.nature.com/tp) 